\title{
New records of lichenicolous fungi from the Gomel Region of Belarus
}

\author{
Andrei Tsurykau ${ }^{1}$, Ave Suija ${ }^{2} \&$ Volha Khramchankova ${ }^{1}$ \\ ${ }^{1}$ Department of Biology, F. Skorina Gomel State University, Sovetskaja st. 104, 246019 Gomel, Belarus. \\ E-mail: tsurykau@gmail.com, hramchenkova@gsu.by \\ ${ }^{2}$ Institute of Ecology and Earth Sciences, University of Tartu, 40 Lai st., 51005, Tartu, Estonia. \\ E-mail: ave.suija@ut.ee
}

\begin{abstract}
Three species of lichenicolous fungi, Roselliniella cladoniae, Taeniolella beschiana and Trichonectria rubefaciens, are reported for the first time for Belarus; two further species, lllosporiopsis christiansenii and Marchandiomyces aurantiacus, are new to Gomel Region, the south-eastern part of Belarus.
\end{abstract}

\section{INTRODUCTION}

Interest in lichenicolous fungi has been constantly rising during the last decades (Lawrey $\&$ Diederich, 2011), but the information about the distribution and habitat preferences of the species remains incomplete. In Belarus lichenicolous fungi are insufficiently known. The first generalizing summary was published in 2011 (Golubkov, 2011a). This paper lists 21 species of lichenicolous fungi [Abrothallus caerulescens Kotte, A. peyritschii (Stein) Kotte, Athelia arachnoidea (Berk.) Jülich, Biatoropsis usnearum Räsänen, Chaenothecopsis epithallina Tibell, Clypeococcum hypocenomycis D. Hawksw., Lichenodiplis lecanorae (Vouaux) Dyko \& D. Hawksw., Libertiella sp., Lichenoconium xanthoriae M. S. Christ., Lichenoconium sp., Muellerella pygmaea (Körb.) D. Hawksw. var. pygmaea Körb., M. ventosicola (Mudd) D. Hawksw., Nectria lichenicola (Ces.) Sacc., Phaeosporobolus usneae D. Hawksw. \& Hafellner, Phoma everniae D. Hawksw., Sphaerellothecium propinquellum (Nyl.) Cl. Roux \& Triebel., Sphinctrina turbinata (Pers.) De Not., Stigmidium congestum (Körb.) Triebel, Tremella cladoniae Diederich \& M. S. Christ., Tremella hypogymniae Diederich \& M. S. Christ, Tremella sp.], six of which (Abrothallus peyritschii, Muellerella pygmaea var. pygmaea, Nectria lichenicola, Phoma everniae, Sphaerellothecium propinquellum and Stigmidium congestum) have been known only according to literature from the beginning of the 20th century (Bachmann \& Bachmann, 1920; Oxner, 1924; Savicz, 1925).

So far four species of lichenicolous fungi (Athelia arachnoidea, Biatoropsis usnearum,
Chaenothecopsis epithallina and Clypeococcum hypocenomycis) have been identified in the Gomel region, south-eastern Belarus (Yurchenko \& Golubkov, 2003; Golubkov, 2011a, 2011b; Tsurykau \& Khramchankova, 2011). Herewith we report five new species for the area, three of which are also new to Belarus.

\section{MATERIAL AND METHODS}

The specimens were collected mainly by the first author during 2003-2012 (Fig. 1). Additionally, the first author examined all Cladonia specimens deposited in the Belarusian Polesye Scientific Herbarium of F. Skorina Gomel State University (GSU) to collect more detailed information on the distribution of Taeniolella beschiana and Roselliniella cladoniae. Most of these specimens were collected in "Pripyatskiy" National Park (PNP). The material was identified mostly by the second author. The specimens were examined using standard methods of microcopy with Nikon Eclipse 80i, Altami CM0745$\mathrm{T}$ and Leica DM750 microscopes. Microscopic structures were studied from hand-cut sections mounted in water. Sizes in parentheses represent minimum and maximum observed values. The number of observations was at least 15. All the voucher specimens are deposited at GSU.

Species distribution in neighbouring countries is provided. As central part of European Russia has not been adequately searched for lichenicolous fungi (Zhurbenko \& Gudovicheva, 2013), the data on species distribution in Russia is also provided for the north-western European 


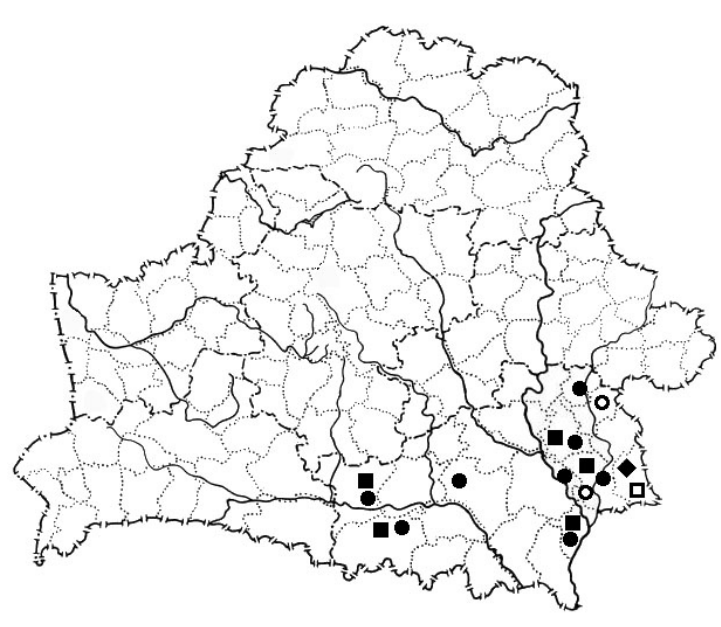

Fig. 1. Locations of finds of studied lichenicolous fungi in Belarus: $\mathbf{\square}$ - Illosporiopsis christiansenii; - Roselliniella cladoniae; - Taeniolella beschiana; 0 - Trichonectria rubefaciens; - Marchandiomyces aurantiacus.

part of the country (see e.g. Zhurbenko, 2007; Stepanchikova et al., 2011). The nomenclature follows Nordin et al. (2011).

The position of the fungus on the lichen thallus is abbreviated as follows: $p=$ podetia, $t$ $=$ lobes of foliose thallus or primary thallus of Cladonia, $\mathrm{s}=$ squamules and phyllocladia, $\mathrm{a}=$ apothecia.

\section{THE SPECIES}

IlLOSPORIOPSis ChristianseniI (B. L. Brady \& D. Hawksw.) D. Hawksw. (Fig 2G \& H)

New to Gomel Region. This species was previously known only in north-western Belarus (Yatsyna, 2011) and the locality in Dobrush district is the second finding in Belarus. Amongst neighbouring countries, the species is known in Latvia (Czarnota \& Kukwa, 2010), Lithuania (Motiejūnaitè, 2002), Poland (Kukwa et al., 2002) and Russia (Leningrad region and Komi Republic) (Kuznetsova, Ahti \& Himelbrant, 2007; Zhurbenko et al., 2012).

Hosts - free living algae, Phaeophyscia orbicularis (Neck.) Moberg (t), Physcia adscendens (Fr.) H. Olivier (t) and Xanthoria polycarpa (Hoffm.) Th. Fr. ex Rieber (t).

Specimens examined - Dobrush district: Korma village, $52^{\circ} 20^{\prime} \mathrm{N}, 31^{\circ} 30^{\prime} \mathrm{E}$, a private small front garden, on Sorbus aucuparia, 19.11.2011, leg. E. Tsukanava.
Marchandiomyces aurantiacus (Lasch) Diederich \& Etayo

New to Gomel Region, the second record for Belarus. The first locality is in north-western Belarus (Yatsyna, 2011). This species is known in Lithuania (Motiejūnaite \& Andersson, 2003) and Poland (Kukwa, 2004).

Hosts - Physcia adscendens (t), P. stellaris (Ach.) Nyl. ( $\mathrm{t}, \mathrm{a})$ and $P$. tenella Bitter $(\mathrm{t})$.

Specimens examined - Dobrush district: Korma village, $52^{\circ} 20^{\prime} \mathrm{N}, 31^{\circ} 30^{\prime} \mathrm{E}$, a private small front garden, on Sorbus aucuparia, 19.11.2011, leg. E. Tsukanava.

Roselliniella Cladoniae (Anzi) Matzer \& Hafellner (Fig. 2C \& D)

New to Belarus. This fungus, occurring sporadically throughout Europe, has been reported in Poland (Kukwa \& Kowalewska, 2007), Lithuania (Motiejūnaitè et al., 2003), Murmansk region of Russia (Zhurbenko\& Alstrup, 2004) and Ukraine (Bielczyk et al., 2005).

Our specimens of $R$. cladoniae are characterized by 25-35 × 10-14 $\mu \mathrm{m}$ non-septate ascospores, which are colourless and halonate when young, but brown and non-halonate when mature. Asci 2-4-spored.

The fungus was found on 21 lichen specimens. The lichens infected with $R$. cladoniae were represented by seven Cladonia species, especially by C. arbuscula ssp. arbuscula (Wallr.) Flot., C. macilenta var. macilenta Hoffm. and C. rangiferina (L.) Weber ex F.H. Wigg., which hosted 16 specimens. $R$. cladoniae is likely to prefer the position on the lichen thallus. The fungus was found twice as often on the podetia than on the primary thallus. The specimens were collected in habitats with varying degree of humidity - from humid Ledum palustre-type pine forest to dry Cladonia-type pine stands. All infected lichen specimens were additionally infected with Taeniolella beschiana (Fig. 2C).

Hosts - Various Cladonia species.

Specimens examined - Buda-Koshelevo district: close to Rudnja-Olhovka village, $52^{\circ} 32^{\prime} \mathrm{N}, 30^{\circ} 22^{\prime} \mathrm{E}$, at the young pine forest margin, on Cladonia cornuta (L.) Hoffm. (p) growing on soil, 21.10.2003, leg. A. Tsurykau; same locality, on C. cenotea (Ach.) Schaer. (p) growing on the bark of pine, 21.10.2003, leg. A. Tsurykau; same locality, pine forest, on C. macilenta var. bacillaris (Ach.) Schaer. (t, p) growing on decaying stump, 15.06.2005, leg. T. Karlovskaja; Gomel district: close to Borets village, pine forest, $52^{\circ} 17^{\prime} \mathrm{N}, 30^{\circ} 57^{\prime} \mathrm{E}$, on C. rangiferina $(\mathrm{p})$ growing on soil, 14.07.1970, leg. A. Paulischava; $1.8 \mathrm{~km} \mathrm{NE}$ of Asovina village, Ledum 

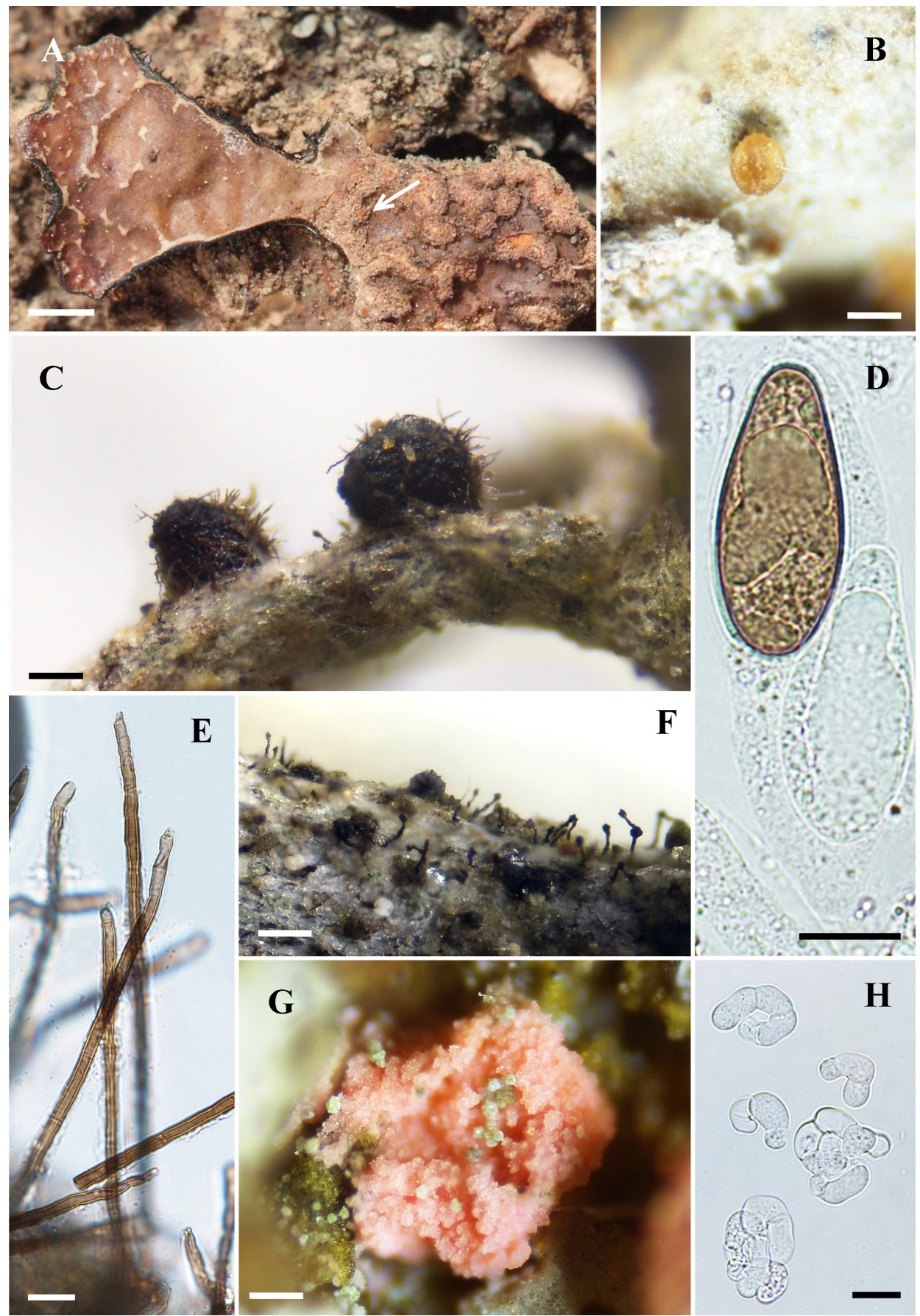

Fig. 2. Lichenicolous fungi. A \& B Trichonectria rubefaciens (2011, Dubrovskaja): A - habitus; B - perithecium. C \& D Roselliniella cladoniae (2011, Tsurykau): C - perithecia, infected with Taeniolella beschiana; D - ascus. E \& F Taeniolella beschiana (2011, Tsurykau): E - conidiophores; F - habitus. G \& H Illosporiopsis christiansenii (2011, Tsukanava): G - sporodochia; H - conidia. Scales: A - $1 \mathrm{~mm}$; B, C, F, G-0.1 mm; D, E, H - $10 \mu \mathrm{m}$. 
palustre-type pine forest $52^{\circ} 13^{\prime} \mathrm{N}, 31^{\circ} 02^{\prime} \mathrm{E}$, on $C$. macilenta $(\mathrm{t})$ growing on the bark of pines, 08.08.2011, leg. A. Tsurykau; $1.9 \mathrm{~km}$ E of Tereshkovichi village, Vaccinium myrtillus-type pine forest, $52^{\circ} 15^{\prime} \mathrm{N}, 30^{\circ}$ $59^{\prime} \mathrm{E}$, on $C$. macilenta (t) growing on the bark of pines, 02.10.2012, leg. A. Tsurykau; ca. $1 \mathrm{~km}$ S of Pribor village, $52^{\circ} 23^{\prime} \mathrm{N}, 30^{\circ} 47^{\prime} \mathrm{E}$, Polytrichum-type pine forest, on C. chlorophaea (Flörke ex Sommerf.) Spreng. (t) growing on pine, 10.10.2012, leg. A. Tsurykau; Le1tchitsy district: PNP, Mlynok forestry, 60 q., 51 ${ }^{\circ} 56^{\prime} \mathrm{N}$, $27^{\circ} 56^{\prime} \mathrm{E}$, on C. arbuscula ssp. arbuscula (p) growing on soil, 18.07.1971, leg. A. Dashuk; same forestry, 60 q., Pleurozium-type pine forest, on C. arbuscula ssp. mitis (Sandst.) Ruoss (p) growing on soil, 19.07.1971, leg. O. Shakhrai and H. Abramava; same forestry, meadow in pine forest with Hieracium and Thymus, on C. arbuscula ssp. mitis (p) growing on soil, 01.06.1974, leg. O. Shakhrai; same forestry, Cladonia-type pine forest, on C. chlorophaea (t, p) growing on brushwood, 01.06.1974, leg. O. Shakhrai; same forestry, Cladoniatype pine forest, on $C$. macilenta $(t, p)$ growing on a stump, 02.07.1975, leg. O. Shakhrai; same forestry, Cladonia-type pine forest, on C. macilenta (t) growing on brushwood, 13.03.1977, leg. V. Prokopenko; same forestry, Cladonia-type pine forest, on C. arbuscula ssp. mitis (p) growing on soil, 24.07.1974, leg. O. Shakhrai; Loyew district: $1.3 \mathrm{~km}$ N of Kawpen village, $51^{\circ} 57^{\prime} \mathrm{N}, 30^{\circ} 39^{\prime} \mathrm{E}$, Cladonia-type pine forest, on C. arbuscula ssp. mitis (p) growing on soil, 09.08.2011, leg. A. Tsurykau; Zhitkovichi district: close to Belev village, pine forest, $52^{\circ} 17^{\prime} \mathrm{N}, 28^{\circ} 02 ' \mathrm{E}$, on C. fimbriata (L.) Fr. $(t, p)$ growing on a stump, 01.02.2006, leg. A. Teikin; PNP, Cladonia-type pine forest, on C. rangiferina $(\mathrm{p})$ growing on soil, 10.06.1975, leg. O. Shakhrai; PNP, Ozerany forestry, $60 \mathrm{q}$., pine forest, $52^{\circ} 00^{\prime} \mathrm{N}, 27^{\circ} 53^{\prime} \mathrm{E}$, on C. rangiferina $(\mathrm{p})$ growing on soil, 25.05.1971, leg. O. Shakhrai; same forestry, 60 q., Vaccinium myrtillus-type pine forest, on C. macilenta (t) growing on soil, 23.07.1974, leg. H. Shvets; PNP, OzeranyMlynok forestry, 106 q., Cladonia-type pine forest, $51^{\circ} 59^{\prime} \mathrm{N}, 27^{\circ} 57^{\prime} \mathrm{E}$, on C. rangiferina $(\mathrm{p})$ growing on soil, 15.03.1977, leg. V. Prokopenko; same forestry, 106 q., Cladonia-type pine forest, on C. arbuscula ssp. mitis (p) growing on soil, 09.05.1977, leg. V. Prokopenko.

\section{TAeniolella Beschiana Diederich (Fig. 2E \& F)} New to Belarus. This hyphomycetous species is the most common fungus inhabiting Cladonia species in Poland (Kukwa et al., 2010). It also known in Franz Josef Land, Russian Karelia and Murmansk region, (Zhurbenko \& Santesson, 1996; Zhurbenko \& Himelbrant, 2002; Zhurbenko \& Alstrup, 2004), and Lithuania (Motiejūnaitè \& Andersson, 2003).

Our specimens of $T$. beschiana are characterized by brown erect conidophores, which carry 9-13 $\times 5 \mu \mathrm{m}$ brown 1-celled conidia.

The fungus was found on 82 lichen specimens.
The lichens infected with Taeniolella beschiana represent 18 species of the genus Cladonia. 55 specimens $(66 \%)$ were growing on C. arbuscula, C. chlorophaea (Flörke ex Sommerf.) Spreng., C. gracilis (L.) Willd., C. macilenta and C. rangiferina. Based on our material, T. beschiana is nonselective to the position on the lichen thallus: the fungus affects both podetia and primary thallus with the same frequency. It was also found on apothecia of C. gracilis and C. squamosa (Scop.) Hoffm. The specimens were collected in habitats with varying degree of humidity - from humid Ledum palustre-type pine forest to dry Cladoniatype pine stands.

Hosts - Various Cladonia species.

Selected specimens examined - Buda-Koshelevo district: close to Rudnja-Olhovka village, $52^{\circ} 32 ' \mathrm{~N}$, $30^{\circ} 22$ 'E, on C. fimbriata (L.) Fr. (t) growing on decaying stump, 21.10.2003, leg. A. Tsurykau; same locality, a clearing in the pine forest, on C. deformis (L.) Hoffm. (t) growing on the bark of birch and on residues, 07.07.2005, leg. A. Tsurykau; same place, on C. rangiferina (p) and C. uncialis (L.) Weber ex F.H. Wigg. (p) growing on soil, 07.07.2005, leg. A. Tsurykau; same locality, near sand-picking pit in pine forest, on $C$. chlorophaea (t, p), C. cornuta $(\mathrm{p})$, C. gracilis $(\mathrm{p}, \mathrm{s}, \mathrm{a})$, C. phyllophora Ehrh. ex Hoffm. (p), C. rangiferina (p) and C. subulata (L.) Weber ex F.H. Wigg. (t) growing on sandy soil, 21.10.2003, leg. A. Tsurykau; same place, on C. cenotea ( $\mathrm{t}, \mathrm{p})$ growing on the bark of pine, 21.10.2003, leg. A. Tsurykau; same locality, at the young pine forest marge, on C. cornuta $(\mathrm{p})$ and $C$. turgida Ehrh. ex Hoffm. (p) growing on soil, 21.10.2003, leg. A. Tsurykau; same locality, in a bog among pine forest, on C. chlorophaea (p) growing on soil, 18.09.2005, leg. A. Tsurykau; same locality, pine forest, on C. macilenta var. bacillaris $(t, p)$ growing on decaying stump, 15.06.2005, leg. T. Karlovskaja; same locality, at the marge of pine forest, on C. arbuscula ssp. mitis (p), C. chlorophaea (t, p), and C. phyllophora (p) growing on soil, 15.06.2005, leg. T. Karlovskaja; close to Klenovitsa village, edge of a mixed forest, $52^{\circ} 36^{\prime} \mathrm{N}, 30^{\circ} 19^{\prime} \mathrm{E}$, on C. cervicornis ssp. verticillata (Hoffm.) Ahti (s) growing on soil, 03.07.2005, leg. A. Tsurykau and N. Timoshenkova; Gomel district: the city of Gomel, Volotova borough, birch grove, $52^{\circ} 28^{\prime} \mathrm{N}$, $31^{\circ} 03^{\prime} \mathrm{E}$, on $C$. cenotea $(\mathrm{t})$ growing on deciduous tree bark, 27.03.2003, leg. I. Kavaliova and I. Hancharova; the city of Gomel, 17 borough, on C. rei Schaer. (t) growing on sandy soil, 29.06.2005, leg. U. Sobchanka; close to Borets village, pine forest, $52^{\circ} 17^{\prime} \mathrm{N}, 30^{\circ} 57^{\prime} \mathrm{E}$, on C. rangiferina $(\mathrm{p})$ growing on soil, 14.07.1970, leg. A. Paulischava; $3 \mathrm{~km}$ SW of Chenki village, close to the Gomel State University's field practice stationary camp, $52^{\circ} 19^{\prime} \mathrm{N}, 30^{\circ} 57^{\prime} \mathrm{E}$, on C. macilenta var. bacillaris (t) growing on decaying lignum, 20.07.2003, leg. I. Kavaliova and I. Hancharova; same place, on C. chlorophaea (t, p) growing on lignum, 20.06.2004, leg. A. 
Tsurykau; $1 \mathrm{~km}$ NE of Tereshkovichi village, Pteridium aquilinum-type pine forest, $52^{\circ} 15^{\prime} \mathrm{N}, 30^{\circ} 58^{\prime} \mathrm{E}$, on $C$. chlorophaea $(\mathrm{t})$ growing on the bark of pines, 03.03.2011, leg. A. Tsurykau; $1.5 \mathrm{~km} \mathrm{NE}$ of Tereshkovichi village, Polytrichum-type pine forest, $52^{\circ} 15^{\prime} \mathrm{N}$, $30^{\circ} 59^{\prime} \mathrm{E}$, on C. macilenta $(\mathrm{t})$ and C. ochrochlora Flörke (t) growing on the bark of pines, 03.03.2011, leg. A. Tsurykau; $1.9 \mathrm{~km}$ E of Tereshkovichi village, Vaccinium myrtillus-type pine forest, $52^{\circ} 15^{\prime} \mathrm{N}, 30^{\circ} 59^{\prime} \mathrm{E}$, on C. macilenta (t) growing on the bark of pines, 02.10 .2012 , leg. A. Tsurykau; $1.8 \mathrm{~km} \mathrm{NE}$ of Asovina village, Ledum palustre-type pine forest, $52^{\circ} 13^{\prime} \mathrm{N}$, $31^{\circ} 02^{\prime} \mathrm{E}$, on $C$. chlorophaea $(\mathrm{t})$ and $C$. macilenta $(\mathrm{t})$ growing on the bark of pines, 08.08.2011, leg. A. Tsurykau; $3.2 \mathrm{~km} \mathrm{NE}$ of Asovina village, Pteridium aquilinum-type pine forest, $52^{\circ} 13^{\prime} \mathrm{N}, 31^{\circ} 03^{\prime} \mathrm{E}$, on $C$. macilenta $(\mathrm{t})$ and $C$. ochrochlora $(\mathrm{t})$ growing on the bark of pines, 08.08.2011, leg. A. Tsurykau; ca. $1 \mathrm{~km} \mathrm{~S}$ of Pribor village, Polytrichum-type pine forest, $52^{\circ} 23^{\prime} \mathrm{N}$, $30^{\circ} 47^{\prime} \mathrm{E}$, on C. chlorophaea (t) and C. coniocraea (Flörke) Spreng. (t) growing on the bark of pines, 10.10.2012, leg. A. Tsurykau; Kalinkovichi district: close to Ozarichi Township, mixed forest, $52^{\circ} 27^{\prime} \mathrm{N}$, $29^{\circ} 16^{\prime} \mathrm{E}$, on C. uncialis (p) growing on soil, 16.08.2004, leg. J. Bachura; Korma district: close to Kljapin village, Polytrichum-type pine forest, near the road, 53 $09^{\prime} \mathrm{N}$, $31^{\circ} 03^{\prime} \mathrm{E}$, on Cladonia $\mathrm{sp}$. (p, a) growing on soil, 12.08.2005, leg. U. Liabedz'ka; Leltchitsy district: PNP, Mlynok forestry, 7 q., Cladonia-type pine forest with Melampyrum nemorosum and Convallaria majalis, $51^{\circ} 56 ' \mathrm{~N}, 27^{\circ} 56^{\prime} \mathrm{E}$, on C. phyllophora $(\mathrm{p})$ growing on soil, w/o date, leg. O. Shakhrai; same forestry, 60 q., on C. arbuscula ssp. arbuscula (p) growing on soil, 18.07.1971, leg. A. Dashuk; same forestry, 60 q., pine forest, on C. rangiferina (p) growing on soil, 1977, leg. H. Abramava; same forestry, 60 q., Pleurozium-type pine forest, on C. arbuscula ssp. mitis (p) growing on soil, 19.07.1971, leg. O. Shakhrai and H. Abramava; same forestry, 61 q., on C. macilenta var. bacillaris (t) and C. cervicornis ssp. verticillata (s) growing on soil, 19.07.1971, leg. J. Klets; same forestry, 72 q., Cladonia-type pine forest, on C. chlorophaea (t) growing on soil, 16.06.1972, leg. O. Shakhrai; same forestry, 106 q., Cladonia-type pine forest, on C. gracilis (p) growing on soil, w/o date, leg. O. Shakhrai; same forestry, 106 q., Pleurozium-type pine forest, on $C$. arbuscula ssp. mitis (p) growing on soil, w/o date, leg. O. Shakhrai; same forestry, Cladonia-type pine forest, on C. arbuscula ssp. mitis (p), C. chlorophaea (t) and C. gracilis $(\mathrm{p})$ growing on soil, 24.07.1974, leg. O. Shakhrai; same forestry, Cladonia-type pine forest, on C. macilenta (t) growing on a stump, 02.07.1975, leg. O. Shakhrai; same forestry, Cladonia-type pine forest, on C. macilenta $(t, p)$ growing on brushwood, 13.03.1977; leg. V. Prokopenko; same forestry, Cytisus-type pine forest, on C. arbuscula ssp. mitis (p) growing on soil, 14.07.1975, leg. A. Radionova; same forestry, Cladonia-type pine forest, on C. rangiferina (p) growing on soil, 06.06.1975, leg. O. Shakhrai; same forestry, pine forest with Hieracium and Nardus, on
C. phyllophora (p) growing on soil, 01.06.1974, leg. O. Shakhrai; same forestry, meadow in pine forest with Hieracium and Thymus, on C. arbuscula ssp. mitis (p) growing on soil, 01.06.1974, leg. O. Shakhrai; same forestry, Cladonia-type pine forest, on C. chlorophaea $(t, p)$ growing on brushwood, 01.06.1974, leg. O. Shakhrai; Loyew district: $1.3 \mathrm{~km}$ N of Kawpen village, Cladonia-type pine forest, $51^{\circ} 57^{\prime} \mathrm{N}, 30^{\circ} 39^{\prime} \mathrm{E}$, on $\mathrm{C}$. arbuscula ssp. mitis (p) and C. gracilis (p) growing on soil, 09.08.2011, leg. A. Tsurykau; same place, on $C$. chlorophaea $(\mathrm{t})$ growing on decaying wood, 09.08.2011, leg. A. Tsurykau; same place, on C. chlorophaea (t) growing on the bark of pines, 09.08.2011, leg. A. Tsurykau; $5 \mathrm{~km}$ W of Kawpenj village, Calluna vulgaris-type pine forest, $51^{\circ} 56^{\prime} \mathrm{N}, 30^{\circ} 35^{\prime} \mathrm{E}$, on C. gracilis (p) and C. squamosa (p, a) growing on soil, 09.08.2011, leg. A. Tsurykau; same place, on C. chlorophaea $(\mathrm{t}, \mathrm{p})$ and $C$. fimbriata $(t, p)$ growing on the bark of pines, 09.08.2011, leg. A. Tsurykau; Retchitsa district: ca. $1.5 \mathrm{~km} \mathrm{E}$ of Borschevka village, pine forest, $52^{\circ} 21^{\prime} \mathrm{N}$, $30^{\circ} 40^{\prime} \mathrm{E}$, on C. macilenta $(\mathrm{t})$ growing on a $1 \mathrm{og}$, 30.09.2006, leg. A. Tsurykau; Zhitkovichi district: close to Belev village, pine forest, $52^{\circ} 17^{\prime} \mathrm{N}, 28^{\circ} 02^{\prime} \mathrm{E}$, on C. fimbriata (t, p) growing on a stump, 01.02.2006, leg. A. Teikin; PNP, Cladonia-type pine forest, on C. rangiferina $(\mathrm{p})$ growing on soil, 10.06.1975, leg. O. Shakhrai; PNP, Ozerany forestry, 50 q., pine forest, $52^{\circ} 00^{\prime} \mathrm{N}, 27^{\circ} 53^{\prime} \mathrm{E}$, on $C$. gracilis $(\mathrm{p})$ growing on soil, 18.06.1971, leg. O. Shakhrai; same forestry, 59 q., Cladonia-type pine forest, on $C$. gracilis $(\mathrm{p})$ growing on soil, 24.07.1975, leg. O. Shakhrai; same forestry, 60 q., pine forest, on C. rangiferina (p) growing on soil, 25.05.1971, leg. O. Shakhrai; same forestry, 60 q., Vaccinium myrtillus-type pine forest, on C. macilenta (t, p) growing on soil, 23.07.1974, leg. H. Shvets; same forestry, on C. cervicornis ssp. verticillata (s), C. deformis (t) and C. macilenta $(\mathrm{t})$ growing on soil, 20.06.1975, leg. O. Shakhrai; same forestry, on C. subulata (t) growing on soil, leg. O. Shakhrai, w/o date; PNP, Ozerany-Mlynok forestry, 85 q., Cladonia-type pine forest, $51^{\circ} 59^{\prime} \mathrm{N}, 27^{\circ} 57^{\prime} \mathrm{E}$, on C. arbuscula ssp. arbuscula (p) growing on soil, 19.03.1977, leg. V. Prokopenko; same forestry, 96 q., Vaccinium vitis-idaea-type pine forest, on C. arbuscula ssp. mitis (p) growing on soil, 10.03.1977, leg. V. Prokopenko; same forestry, 98 q., Vaccinium vitis-idaea-type pine forest, on C. chlorophaea $(\mathrm{t})$ growing on brushwood, 15.03.1977, leg. V. Prokopenko; same forestry, 106 q., Cladoniatype pine forest, on C. rangiferina $(\mathrm{p})$ growing on soil, 15.03.1977, leg. V. Prokopenko; same forestry, 106 q., Cladonia-type pine forest, on C. arbuscula ssp. mitis (p) growing on soil, 09.05.1977, leg. V. Prokopenko; PNP, Pererov forestry, 18 q., Cladonia-type pine forest, $52^{\circ} 02 ' \mathrm{~N}, 27^{\circ} 58^{\prime} \mathrm{E}$, on C. cervicornis ssp. verticillata $(\mathrm{t}, \mathrm{p})$ growing on soil, 20.07.1974, leg. O. Shakhrai; same forestry, 23 q., Polytrichum-type pine forest, on C. gracilis (p) growing on soil, 05.07.1973, leg. L. Parukova; PNP, Rychev forestry, 64 q., Cladonia-type pine forest, $51^{\circ} 59^{\prime} \mathrm{N}, 27^{\circ} 42 ' \mathrm{E}$, on C. arbuscula ssp. mitis (p) growing on soil, 17.07.1972, leg. O. Shakhrai. 
Trichonectria Rubefaciens (Ellis \& Everh.) Diederich \& Schroers (Fig. 2A \& B)

New to Belarus. This pathogenic fungus is known from Lithuania (Motiejūnaitè at al., 2012), Russia, Leningrad region (Kuznetsova et al., 2012), Poland (Kukwa et al., 2010) and Ukraine (Pirogov, 2011).

Our specimens are characterized by having 1-septate, 14-15 $\times 3 \mu \mathrm{m}$ colourless ascospores. Perithecia orange, superficial, ca. $0.1 \mathrm{~mm}$ in diameter.

Host - Parmelia sulcata Taylor (t).

Specimens examined - Chechersk district: vicinity of Gaijok village, $52^{\circ} 58^{\prime} \mathrm{N}, 31^{\circ} 08^{\prime} \mathrm{E}$, at the edge of pine forest, on the bark of Betula pendula, 16.09.2011, leg. E. Dubrovskaja; Gomel district: $3 \mathrm{~km} \mathrm{SW}$ of Chenki village, close to the fence at Gomel State University's field practice stationary camp, $52^{\circ} 19^{\prime} \mathrm{N}, 30^{\circ} 57^{\prime} \mathrm{E}$, on bark of B. pendula, 25.06.2012, leg. A. Tsurykau.

\section{ACKNOWLEDGEMENTS}

Dr Piret Lõhmus (Tartu) is warmly thanked for financial support of the visit of A. T. to Tartu, which promoted this study. Dr Jurga Motiejūnaite (Vilnius) provided information on the distribution of the examined species in Lithuania. Dr Robert Szava-Kovats is thanked for correcting the language. Prof M. P. Zhurbenko (Saint-Petersburg) is thanked for his information and suggested amendments which have improved the paper.

\section{REFERENCES}

Bachmann, E. \& Bachmann, F. 1920. Litauische Flechten. Hedwigia 61(6): 321-342.

Bielczyk, U., Bylińska, E., Czarnota, P., Czyżewska, K., Guzow-Krzemińska, B., Hachułka, M., Kiszka, J., Kowalewska, A., Krzewicka, B., Kukwa, M., Leśniański, G., Śliwa L. \& Zalewska A. 2005. Contribution to the knowledge of lichens and lichenicolous fungi of Western Ukraine. Polish Botanical Journal 50(1): 39-64.

Czarnota, P. \& Kukwa, M. 2010. New and noteworthy lichenized and lichenicolous fungi from Latvia. Botanica Lithuanica 16(1): 21-27.

Etayo, J. 1998. Some hypocrealean lichenicolous fungi from Southwest Europe. Nova Hedwigia 67(3-4): 499-509.

Golubkov, V. V. 2011a. Annotated checklist of lichenicolous fungi of Belarus. Botany: Research 40: 295-306. (In Russian).

Golubkov V. V. 2011b. Lichen biota of "Pripyatsky" National park (in Russian). Minsk. 192 pp.

Kukwa, M. 2004. New or interesting records of lichenicolous fungi from Poland II. Species mainly from northern Poland. Herzogia 17: 67-75.
Kukwa, M., Czarnota, P. \& Perz, P. 2010. New or interesting records of lichenicolous fungi from Poland VIII. Herzogia 23: 111-119.

Kukwa, M. \& Kowalewska, M. 2007. New or interesting records of lichenicolous fungi from Poland V. Species mainly on Cladonia. Herzogia 20: 199-207.

Kukwa, M., Motiejūnaitè, J., Rutkowski, P. \& Zalewska, A. 2002. New or interesting records of lichenicolous fungi from Poland I. Herzogia 15: 129-139.

Kuznetsova, E., Ahti T. \& Himelbrant, D. 2007. Lichens and allied fungi of the Eastern Leningrad Region. Norrlinia 16: 1-62.

Kuznetsova, E. S., Motiejūnaitè, J, Stepanchikova, I. S., Himelbrant, D. E. \& Czarnota, P. 2012. New records of lichens and allied fungi from the Leningrad Region, Russia. III. Folia Cryptogamica Estonica 49: 31-37.

Lawrey, J. D. \& Diederich, P. 2011. Lichenicolous fungi - worldwide checklist, including isolated cultures and sequences available. http://www. lichenicolous.net.

Motiejūnaitè, J. 2002. Diversity of lichens and lichenicolous fungi in the transboundary region of Marijampole district (southern Lithuania). Botanica Lithuanica 8(3): 277-294.

Motiejūnaitè, J. \& Andersson, L. 2003. Contribution to the Lithuanian flora of lichens and allied fungi. Botanica Lithuanica 9(1): 71-88.

Motiejūnaitè, J., Berglund, T., Czarnota, P., Himelbrant, D., Högnabba, F., Konoreva, L. A., Korchikov, E. S., Kubiak, D., Kukwa, M., Kuznetsova, E., Leppik, E., Lõhmus, P., Prigodina Lukošienè, I., Pykälä, J., Stončius, D., Stepanchikova, I., Suija, A., Thell, A., Tsurykau, A. \& Westberg, M. 2012. Lichens, lichenicolous and allied fungi found in Asveja Regional park (Lithuania). Botanica Lithuanica 18(2): 85-100.

Motiejūnaitè, J. \& Czyżewska, K. 2008. Additions to the biota of lichens and lichenicolous fungi of Poland, with a note on Lecania prasinoides in Eastern and Central Europe. Polish Botanical Journal 53(2): 155-162.

Motiejūnaitè, J., Kukwa, M., Czarnota, P., PrigodinaLukošienè, I., Himelbrant, D., Kuznetsova, E. \& Kowalewska, A. 2003. Lichens and allied fungi collected during the 15th Symposium of Baltic Mycologists and Lichenologists in Birštonas, Lithuania. Botanica Lithuanica 9(2): 109-119.

Nordin, A., Moberg, R., Tønsberg, T., Vitikainen, O., Dalsätt, Å., Myrdal, M., Snitting, D. \& Ekman, S. 2011. Santesson's Checklist of Fennoscandian Lichen-forming and Lichenicolous Fungi. Ver. April 29, 2011 - http://130.238.83.220/santesson/ home.php

Oxner, A. N. 1924. Beiträge zur Flechtenflora Wissrusslands. Bulletin du Jardin Botanique de Kieff 1: 27-36. (in Russian).

Pirogov, M. V. 2011. Nectriopsis rubefaciens (Ellis \& Everh.) M. S. Cole \& D. Hawksw. (Bionectriaceae) a new species of lichenicolous fungus for Ukraine. Ukrainian Botanical Journal 68(1): 127-128. 
Savicz, V. P. 1925. Die Resultate lichenologischer Untersuhungen in Weissrussland im Jahre 1923. Mémoires de l'institut agronomique et forestier d'état de la Bélarussie 4: 1-33 (in Russian).

Stepanchikova, I. S., Himelbrant, D. E., Kukwa, M. \& Kuznetsova, E. S. 2011. New records of lichens and allied fungi from the Leningrad Region, Russia. II. Folia Cryptogamica Estonica 48: 85-94.

Tsurykau, A. \& Khramchankova, V. 2011. Lichens from Gomel region: a provisional checklist. Botanica Lithuanica 17(4): 157-163.

Yatsyna, A. 2011. The first contribution to lichens, lichenicolous and allied fungi from Braslav Lakes National Park (NW Belarus). Botanica Lithuanica 17(4): 177-184.

Yurchenko, E. O. \& Golubkov, V. V. 2003. The morphology, biology, and geography of a necrotrophic basidiomycete Athelia arachnoidea in Belarus. Mycological Progress 2(4): 275-284.

Zhurbenko, M. P. 2007. The lichenicolous fungi of Russia: geographical overview and a first checklist. Mycologia Balcanica 4: 105-124.
Zhurbenko, M. P. \& Alstrup, V. 2004. Lichenicolous fungi on Cladonia mainly from the Arctic. Symbolae Botanicae Upsalienses 34(1): 477-499.

Zhurbenko M. P. \& Gudovicheva A. V. 2013. Zwackhiomyces echinulatus and other lichenicolous fungi from Tula Region of Russia. Mycology and phytopathology 47(1): 19-20. (in Russian).

Zhurbenko, M. P., Hermansson J. \& Pystina T. N. 2012. Lichenicolous fungi from the Komi Republic of Russia. II. Folia Cryptogamica Estonica 49: 89-91.

Zhurbenko, M. P. \& Himelbrant, D. E. 2002. Lichenicolous fungi from the Kandalaksha Gulf, Karelia Keretina, Russia. Folia Cryptogamica Estonica 39: 51-59.

Zhurbenko, M. P. \& Santesson, R. 1996. Lichenicolous fungi from the Russian Arctic. Herzogia 12: 147-161. 
Folia Cryptog. Estonica 